\title{
Simulation methodology for performance and safety evaluation of human-industrial robot collaboration workstation design
}

\author{
Fredrik Ore $^{1,2} \cdot$ Bhanoday Vemula $^{1}$ (C) $\cdot$ Lars Hanson $^{2,3,4} \cdot$ Magnus Wiktorsson $^{1} \cdot$ Björn Fagerström $^{1}$
}

Received: 23 March 2018 / Accepted: 28 June 2019 / Published online: 17 July 2019

(c) The Author(s) 2019

\begin{abstract}
There is a strong interest in the scope of human-industrial robot collaboration (HIRC) in manufacturing industry for greater flexibility and productivity. However, HIRC in manufacturing is still in its infancy; industrial practitioners have many apprehensions and uncertainties concerning the system's performance and human operators' safety. Therefore, there is a need for investigations into design processes and methods to make sure the designed HIRC workstations successfully meet design guidelines on system performance, human safety and ergonomics for practical industrial applications. This research proposes a HIRC workstation design process. The novelty of this design process is the methodology to evaluate the HIRC workstation design alternatives by considering both performance and safety characteristics through computer-based simulations. As a proof of concept, the proposed HIRC design process is applied on an industrial manufacturing case from a heavy-vehicle manufacturing company.
\end{abstract}

Keywords Human industrial robot collaboration $\cdot$ Safety $\cdot$ Ergonomics $\cdot$ Collision model $\cdot$ Performance evaluation $\cdot$ Risk assessment

\section{Introduction}

There is a growing interest in making humans and industrial robots share their working environments, sometimes even allowing direct physical contact between the two in order to make them work collaboratively on the same task (Fryman and Matthias 2012; Zinn et al. 2004; Tsarouchi et al. 2016). This concept is described as "a dream combination of human flexibility and machine efficiency" (Tan et al. 2009, p. 29). The goal is to combine human intelligence and flexibility with industrial robotic strength, endurance and accuracy (Helms et al. 2002; Krüger et al. 2005) to build workstations

Bhanoday Vemula

bhanoday29@gmail.com

1 School of Innovation, Design and Engineering, Mälardalen University, 63105 Eskilstuna, Sweden

2 Scania CV AB, Global Industrial Development, 15187 Södertälje, Sweden

3 School of Engineering Science, University of Skövde, 54128 Skövde, Sweden

4 Department of Industrial and Materials Science, Chalmers University of Technology, 41296 Göteborg, Sweden with higher productivity and lower ergonomic loads (Krüger et al. 2009).

However, human safety is a prerequisite for successful implementation of applications based on human-industrial robot collaboration (HIRC) in the manufacturing industries. The traditional view on human safety in robot automation is to separate robots from humans with physical fences. HIRC systems imply fenceless workstations, thus requiring new robust safety measures to guarantee human safety. ISO 10218 gives a brief description of basic safety requirements for four types of HIRC operations: (1) safety-rated monitored stop, (2) hand guiding, (3) speed and separation monitoring and (4) power and force limiting (ISO: ISO 10218-2:2011 2011). HIRC workstations designed for practical manufacturing operations often include a combination of these different types of HIRC operations. Therefore standardised methods of reduction of risk associated with these different HIRC operations need to be holistically considered during planning and subsequent evaluation of the HIRC workstation design (ISO: ISO 10218-2:2011 2011).

Where there is a possibility of human-robot physical contact, there is an inherent risk of causing sensory pain or injury to the human. Therefore, no matter what safety considerations are incorporated by the robot manufacturers 
in the selected industrial robot system and to what extent its performance capabilities are limited during the collaborative applications, HIRC workstations in general cannot be deemed absolutely safe for human co-workers and risk estimation becomes essential during the design process of HIRC applications. Whenever there are unacceptable risks associated with the human operator's safety and wellbeing, design priority given to realise the safety objectives should always supersede the priority given to realise the performance objectives in the manufacturing industry (Michalos et al. 2015). However, too many performance limitations can reduce the productivity to an extent where the very purpose of implementing the HIRC workstation becomes questionable. In this context, it is very important to evaluate both the performance and the inherent safety characteristics associated with the HIRC workstation during the design and development stages. Still, in early phases of production design it is difficult to evaluate these characteristics due to the absence of a physical workstation. This highlights the need for virtual workstations evaluated through simulation software in order to facilitate a bettergrounded production investment decision.

In addition, there is also a wide range of design choices during the HIRC workstation design process in terms of industrial robot hardware and performance trade-off scenarios in favour of hard safety requirements. Finding the optimal combination of performance and safety objectives can be an iterative and time-consuming process. Hence, carrying out performance and safety evaluation of HIRC workstation designs using computational efficient simulation models can facilitate evaluation of several operational scenarios.

Previous research studies have been carried out in the area of simulation-based HIRC workstation evaluation. These studies were carried out with an emphasis on either operational performance requirements or human safety aspects in a fenceless environment. Tsarouchi et al. (2016) present one example of a performance evaluation covering multiple characteristics, floor space, robot reachability, ergonomics and investment cost in a HIRC workstation, and Park et al. (2011) and Oberer et al. (2007, 2010) present methods to evaluate collision forces between human and robot as a measure of human safety. However, there is a gap in simulation-based evaluation methods that can be used to consider both the operational performance and human safety during the early phases of a HIRC workstation design process. To address this gap, this study introduces a developed simulation-based HIRC workstation design process. The novelty of this process is the methodology to evaluate the HIRC workstation design alternatives by considering both safety and performance characteristics.

In this research, the proposed HIRC workstation design process is applied on a practical industrial case to demonstrate how human safety and performance characteristics of a specific HIRC design can be evaluated.

\section{Related work}

\subsection{HIRC design process}

In general, design processes include three main stages: analysis, synthesis and evaluation, commonly referred to as the ASE process (Luckman 1967; Braha and Maimon 1997). These stages are defined by Luckman (1967) as follows: analysis contains collection and classification of all the relevant information, including objective and constraints of the design problem, synthesis covers the formulation of potential solutions and evaluation covers the assessment of the potential solutions to select the most appropriate one. ASE processes are more efficient if they are iterated multiple times throughout the design process.

ASE-based design methods for manufacturing workstations are commonly used in the research area of manufacturing systems design (Wu 1994; Bellgran 1998). However, very few research studies have been targeted at methods for designing HIRC workstations. These studies were mainly limited to the work task allocation problem to optimally share manufacturing operations between human and robot. One example of this is a method to qualitatively compare suitability between manual and robotic task allocation together with economic profitability (Pini et al. 2015). Another method based on multi-objective optimisation techniques to choose a suitable task allocation considering assembly time and economic cost was proposed by Chen et al. (2011). A similar task allocation method for HIRC design that considers mean flowtime and utilisation costs was proposed by Tsarouchi et al. (2017).

In recent years, several research activities in the development of HIRC simulation software have been undertaken. Advanced digital human modelling (DHM) software is often used in these types of tools to reproduce human behaviour in HIRC systems; the virtual human models in this software are named 'digital manikins' or only 'manikins'. Robot simulation functionality is also required. Tsarouchi et al. (2016) presented a study where the Process Simulate software is used to present multiple design solutions to a HIRC workstation layout problem. IPS-HIRC is another software that enables simultaneous human and robot motions on an object in a HIRC workstation (Ore et al. 2015).

\subsection{Performance evaluation of HIRC design}

Two of the key anticipated benefits of HIRC workstations compared with manual ones are increased productivity and improved ergonomics for the operators (Krüger et al. 2009). 
Productivity is generally measured in terms of total operation time of the manufacturing task in the workstation, which in HIRC systems includes robotic, manual and collaborative times. Robotic time can be extracted from simulation tools while manual time can be predicted using predetermined motion time systems (PMTS). There are a number of PMTS, where the first widespread standard was the Methods-Time Measurement (MTM) system developed in the USA in the 1940s (Laring et al. 2002).

Ergonomics is a wide field comprising many factors (e.g., lighting, psychological and physical stress, sound) that affect human well-being and system performance. However, in this research ergonomics is considered only in terms of physical stress due to biomechanical loads on the human operator. Existing biomechanical assessment methods can be divided into three categories, self-reports, observational methods and direct measurements (David 2005). The latter two are possible to use in geometric simulations. Through digital manikins performing a motion the joint angles can be extracted (Thomas et al. 2016). The numerical values of the joint angles can then be used to quantify the risk of musculoskeletal injuries when performing a manufacturing task (Vignais et al. 2017). These observational methods are commonly used to analyse biomechanical load on physical operators performing tasks in industry (Genaidy et al. 1994). The methods are developed to analyse a work operation by evaluating postures of the human body when performing tasks. A number of different posture observation methods are used in the industrialised environment, of which OWAS (Karhu et al. 1977), RULA (McAtamney and Corlett 1993) and REBA (Hignett and McAtamney 2000) are some of the most frequently used (Kee and Karwowski 2007).

The total cost of a HIRC workstation is another important performance metric. Krüger et al. (2009) exemplified this with the cost of purchase and installation of a robot compared with the personal saving that the robot generates. Another simple example of cost calculation is presented by Tsarouchi et al. (2016), where an investment cost criterion is calculated for each HIRC design alternative.

\subsection{Evaluation of safe human-robot impact behaviour during HIRC}

In general, injury is not an option in robotics. The robot should not injure a human, especially in industrial use (ISO: ISO/TS 15066:2016 2016). Therefore, based on the pioneering work by Yamada et al. (Yamada et al. 1997), significant research efforts have been directed in recent times towards risk assessments and risk reduction of industrial robot systems and HIRC workstation designs by considering human pain tolerance as a criterion for safe robot impact behaviour (Povse et al. 2010; Behrens and Elkmann 2014). Lately, several researchers have carried out biomechanical investigations in order to correlate physical impact quantities such as contact forces, surface pressures and energy density transmitted into the human body with respect to the onset of pain sensations and occurrence of contusion or bruise (Yamada et al. 1997; Povse et al. 2010; Behrens and Elkmann 2014). As of now, there is no clear view in the research community as to what impact quantity can be most relevantly used as an indicator of the occurrence of contusion or pain sensation (Behrens and Elkmann 2014). Nevertheless, the current state of industrial practice for evaluating safe human-industrial robot impact behaviour is specified in ISO 15066 (ISO: ISO/TS 15066:2016 2016). According to this, specific threshold values of contact forces and pressures are set for different regions of the human body, which when exceeded will indicate unsafe human-industrial impact behaviour.

Therefore, there arises a need to quantify contact forces and energy density (energy deposited per area on the human body) in different regions of the human body by an industrial robot system during a HIRC application. Such quantifications are mainly carried out in the following four ways: (1) experimental collision testing using standard automotive crash testing equipment (Haddadin et al. 2007, 2009), (2) by using sensory test-bed setups with predefined inertial and compliance properties (Dagalakis et al. 2016; Matthias et al. 2014), (3) simulated crash tests using explicit finite element (FE) solvers (Oberer and Schraft 2007) and (4) by using models based on the compliant contact force (CCF) modelling approach based on the Hertz contact theory (Park et al. 2011; Wassink and Stramigioli 2007).

Considering the highly iterative nature of the HIRC workstation design process, simulation-based collision tests are more appropriate than physical crash tests, and thus simulation methods based on FE and CCF modelling approaches are preferred. In recent times, great progress has been made in developing human models such as THUMS and HUMOS for FE simulations, which are intended for simulating crash tests in the automobile industry (Maeno and Hasegawa 2001; Robin 2001). These FE models not only constitute the geometrical contours of the human body but also consider complex material properties such as the nonlinear viscohyperelastic, anisotropic and nonhomogeneous nature of the human's biological soft tissues. Designers can adopt these models and achieve reliable estimates of the impact quantities during the collision tests between the human and industrial robots. However, since the boundary conditions, such as the contact area between the two colliding bodies, change over time, a time-consuming nonlinear dynamic analysis is required, thus making the FE approach a computationally intensive process. Therefore, several researchers have shown interest in developing CCF models due to their time effectiveness (Park et al. 2011; Wassink and Stramigioli 2007; Flores et al. 2006; Hertz 1881). The CCF models can 


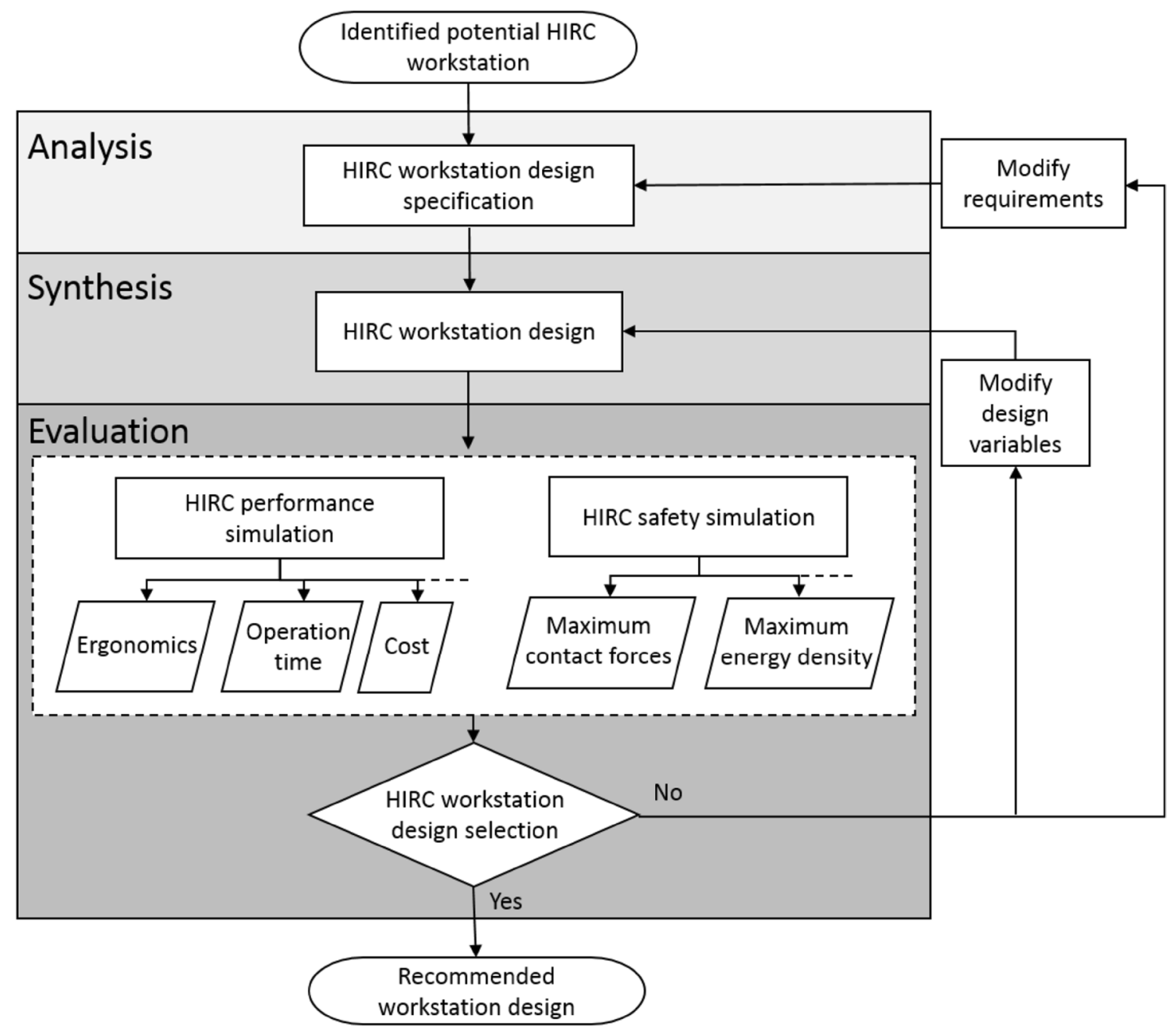

Fig. 1 Proposed HIRC workstation design process

replace the time-consuming FE simulation models where timely evaluation of the impact quantities is of significance.

\section{Proposed HIRC workstation design method}

In this section the proposed HIRC workstation design process based on the general ASE process is presented. The different design activities corresponding to each of the sub processes are listed and briefly described in this section.

\subsection{Method to acquire the design method}

The proposed HIRC workstation design process is based on the general ASE process and the HIRC competence of the authors. The authors have many years of research as well as practical HIRC and workstation design expertise.

\subsection{HIRC workstation design method}

This HIRC process includes the main processes of analysis, synthesis, evaluation and sub process (Fig. 1). The process is generic and can be used to design any HIRC system. It requires simulation software that can enable quantitative evaluation of performance and safety-related characteristics.

The input to start the HIRC workstation design process is an identified workstation, existing or in the planning phases, that might benefit from an industrial robot performing some work tasks. The identified workstation must have geometric boundaries on the shop floor as well as defined functional limitations in terms of what to produce.

\subsubsection{Analysis}

The analysis phase includes the following design activities, which need to be executed sequentially. 
A. Investigation of a specific manufacturing workstation of interest from a HIRC workstation design perspective.

B. Identification of a set of operations, performance and safety requirements based on (A).

C. Task division between human and industrial robot. One method of this is presented in (Ore et al. 2016).

D. Identification of relevant HIRC workstation design parameters. These parameters can be related to workstation layout dimensions, industrial robot system, robot speed limits, anthropometrics of the operators, robot gripper design, geometrical robot paths and handover positions between human and robotic tasks.

\subsubsection{Synthesis}

The synthesis phase includes the following design activities, which need to be executed sequentially after the analysis process.

E. Selection of appropriate design parameters identified in (D) as design variables to facilitate multiple design variants.

F. Allocation of appropriate dimensions and values to all design parameters.

\subsubsection{Evaluation}

The main novelty of the design process presented in this study lies in the evaluation of safety as well as performance of the HIRC workstation design through computer-based simulation software. This process includes the following design activities:

G. The performance capabilities of the HIRC workstation design are measured in terms of ergonomics, operation time, cost and any other performance measures of interest in the specific case. And the safety simulation consider contact forces, energy density and other measures of interest. The simulation software has to be able to output quantitative data of these measures.

H. Finally the HIRC workstation design is evaluated and a decision is made based on the performance and safety characteristics. If not, the ASE process needs to be iterated by modifying the design variables (as a first priority) and the requirements specifications (as a second priority) (see iteration loops in Fig. 1).

\section{Application of proposed HIRC workstation design method}

The HIRC design process proposed is applied in an industrial setting and presented below. Before this is done, the specific software and safety metrics are described.

\subsection{Method for acquiring performance measures}

During application of the HIRC workstation design process, a research version of the simulation software IPS (Industrial Path Solutions) was used (IPS 2017). This software combines the DHM and robotic parts of the IPS software and is named IPS-HIRC in this paper. The DHM part of the software has been developed to automatically predict human motions to suit the virtual environment (Högberg et al. 2016). It uses mathematical algorithms to normalise the loads on each joint of the manikin in order to determine a posture with the most favourable biomechanical load (Bohlin et al. 2012). The postures are then combined to a motion. The robotic part of the software also uses mathematical algorithms to optimise robot trajectories (Segeborn et al. 2014). This IPS-HIRC software was used to evaluate the performance measures of operation time and biomechanical load in the industrial case presented.

\subsubsection{Time evaluation}

Total operation time includes the duration of completing the robotic, the human and the collaborative tasks. Robotic operation time was calculated in the simulation software. It uses robotic joint velocities from the robot data sheet to create optimum robotic motion paths and returns the total robotic handling time (Segeborn et al. 2014). Human times are analysed through the predetermined motion time systems (PMTS) methods-time measurement (MTM) method (Maynard et al. 1948) automatically in the IPS-HIRC software. Based on the digital manikin and geometric positions defined in the simulation software, distances to move joints of the manikin are calculated, and these result in specific operation times to perform the motions. The collaborative times are calculated as human or robotic time, depending on who controls the speed of the task.

\subsubsection{Biomechanical load evaluation}

The observation technique rapid upper limb assessment (RULA) is one of the most cited observational methods used to examine the biomechanical load (Vignais et al. 2017). Therefore, it is used in the IPS-HIRC software to estimate the biomechanical load on the digital manikins. RULA includes an assessment worksheet that is used to analyse individual 
Table 1 RULA values and their interpretations of risk on humans (McAtamney and Corlett 1993)

\begin{tabular}{ll}
\hline RULA value & Interpretations of RULA value \\
\hline $1-2$ & Acceptable posture, workstation \\
$3-4$ & Further investigations of the posture are needed and changes of the \\
& workstation might be required \\
$5-6$ & Investigations and changes of the workstation are required soon \\
7 & Immediate investigations and changes of the workstation are needed \\
\hline
\end{tabular}

manikin poses in a work task (Genaidy et al. 1994). Each manikin motion consists of a number of poses, and each of the poses is automatically analysed through the RULA method. RULA quantifies the risk of musculoskeletal injuries on a human posture on a scale from one to seven, where a high score represents a high risk of future injuries. The RULA values represent risks on the human of future musculoskeletal disorders; the recommendations for each value are given in Table 1 (McAtamney and Corlett 1993). A time-weighted mean RULA score is used as a metric for the biomechanical load evaluation.

\subsection{Method for acquiring safety measures}

Relevant impact quantities such as collision forces and energy deposited in the human body need to be calculated during the human-robot collision in order to measure the safety characteristics of the HIRC workstation. Considering the time-effectiveness of the CCF modelling approach, it has been adopted in this research to calculate and subsequently formulate the relevant safety measures for evaluating safe human-robot impact behaviour in HIRC workstations. The human-robot collision model used in this research is based on the authors' previous research work reported in Vemula et al. (2018). It can be noted that the collision model mentioned was verified based on the experimental collision data reported by Behrens et al. (Behrens and Elkmann 2014). An overview of the collision model is given in this section.

The normal collision process between robot and human body is expressed as a single degree of freedom dynamic system. When the collision process begins, the local deformation $(\delta)$ between the robot and the human body is zero and the impact velocity $\left(\mathrm{V}_{\mathrm{i}}\right)$ is defined as the relative difference between the velocities of the robot and the human body at impact. These initial conditions can be used for expressing the contact force $F(\delta)$ using the equation of motion in terms of the local deformation ( $\delta)$, Eq. (1).

$\ddot{\delta}=\frac{\mathrm{F}(\delta)}{\mathrm{M}}$

$M$ represents equivalent mass, which is expressed for the unconstrained transient contact scenario in which the human body can retract from impact by a moving robot body, Eq. (2). The case of the constrained transient contact scenario, where the human body part is constrained and cannot retract from impact by a moving robot body, is given in Eq. (3).

$M=\left(\frac{1}{M_{H}}+\frac{1}{M_{R}}\right)^{-1}$

$\mathrm{M}=\mathrm{M}_{\mathrm{R}}$

$M_{R}, M_{H}$ are the reflective masses of the robot and the human body, respectively. For a given multi-link robot manipulator, $\mathrm{M}_{\mathrm{R}}$ at the point of impact on the robot body in the operational space can be accurately calculated for a specific set of joint space parameters $(q)$ from the kinetic energy matrix $M_{C}$ in the operational space (x) as given in Eq. (4) (Khatib and Burdick 1987). Based on the decomposition of the kinetic energy matrix inverse, the robot's reflective mass $\left(M_{R}\right)$, which is a scalar value, can be obtained. $M_{R}$ is the robot's mass perceived at the end-effector given a force in $\mathrm{u}$ direction as given in Eq. (4) (Khatib and Burdick 1987).

$\mathrm{M}_{\mathrm{C}}=\left(\mathrm{J}(\mathrm{q}) \mathrm{M}^{-1}(\mathrm{q}) \mathrm{J}^{\mathrm{T}}(\mathrm{q})\right)^{-1}$
$\mathrm{M}_{\mathrm{R}}(\mathrm{x})=\left[\mathrm{u}^{\mathrm{T}} \mathrm{M}_{\mathrm{C}}^{-1}(\mathrm{q}) \mathrm{u}\right]^{-1}$

$\mathrm{J}(\mathrm{q})$ is the Jacobian matrix, $\mathrm{M}(\mathrm{q})$ is the symmetric positive definite mass matrix and $u$ is the unit vector. On the other hand, data related to the $\mathrm{M}_{\mathrm{H}}$ values corresponding to different body regions are adopted from Haley (1988), and are shown in Table 2 for reference. These are averaged mass values collected from different human subjects of various age groups, gender and anthropometric diversity.

Subsequently, the physical collision between the robot and the human body can be expressed as a linear springdamper system, which can be used as a simplified modelling approach to represent the physical nature of the energy transferred between them during the contact process. In such models, contact force can be represented as a contact force law with a linear viscous-elastic term as shown in Eq. (5) (Hertz 1881; Hunt and Crossley 1975).

$\mathrm{F}=\mathrm{K} \delta+\mathrm{C} \dot{\delta} \delta$

$\mathrm{K}$ represents the contact stiffness, $\mathrm{C}$ is the damping coefficient and $\dot{\delta}$ is the rate of deformation during the impact process. Several researchers have proposed various expressions 
Table 2 Effective masses (Högberg et al. 2016) and spring constants (Segeborn et al. 2014) of different human body regions

\begin{tabular}{lll}
\hline Body region & $\begin{array}{l}\text { Effective spring con- } \\
\text { stant }(K)(\mathrm{N} / \mathrm{mm})\end{array}$ & $\begin{array}{l}\text { Effective } \\
\text { mass }\left(M_{\mathrm{H}}\right) \\
(\mathrm{kg})\end{array}$ \\
\hline Skull and forehead & 150 & 4.4 \\
Face & 75 & 4.4 \\
Neck & 50 & 1.2 \\
Back and shoulders & 35 & 40 \\
Chest & 25 & 40 \\
Abdomen & 10 & 40 \\
Pelvis & 25 & 40 \\
Upper arms and elbow joints & 30 & 3 \\
Lower arms and wrist joints & 40 & 2 \\
Hands and fingers & 75 & 0.6 \\
Thighs and knees & 50 & 75 \\
Lower legs & 60 & 75 \\
\hline
\end{tabular}

for $\mathrm{C}$ as a function of coefficient of restitution $\left(\mathrm{C}_{\mathrm{R}}\right)$ (Flores et al. 2006; Hunt and Crossley 1975), resulting in many variants of contact force models, which can account for dissipative forces. The proposed study aims at evaluating the highly inelastic collisions for the worst-case considerations. Therefore, the formulation given in Eq. (6) for expressing the damping coefficient is adopted from Flores et al. (2006), since it has been demonstrated to be reliable for the impact cases with smaller $C_{R}$ values.

$\mathrm{C}=\frac{8 \mathrm{~K}\left(1-\mathrm{C}_{\mathrm{R}}\right)}{5 \mathrm{C}_{\mathrm{R}} V_{i}}$

From (5) and (6), the contact force $\left(\mathrm{F}_{\mathrm{C}}\right)$ can be expressed as (7).

$\mathrm{F}_{\mathrm{C}}=\mathrm{K} \delta\left[1+\frac{8\left(1-\mathrm{C}_{\mathrm{R}}\right)}{5 \mathrm{C}_{\mathrm{R}}} \frac{\dot{\delta}}{\mathrm{V}_{\mathrm{i}}}\right]$

$\mathrm{K}$ represents the effective spring constant corresponding to a specific human body region. The values of $\mathrm{K}$ for different body regions are experimentally derived for different body regions in (Unfallversicherung 2009), which can be seen in Table 2. These compression constants corresponding to different body regions are reported in (Unfallversicherung 2009); the data are derived from averaged values measured from several human subjects of different gender and anthropometric diversity. 139 body dimensions of standing and seated males obtained by traditional anthropometric methods and stereo-photographic techniques were used to derive data corresponding to the effective masses in (Haley 1988). These data are currently used in the force- and pressure-measuring devices intended to carry out human-robot collision-based risk analysis (Dagalakis et al. 2016; Huelke and Ottersbach
2012) and hence adopted in this research considering the current state of industrial practice.

Maximum threshold values of acceptable contact forces and pressures for safe human-robot impact behaviour are standardised for different human body regions (ISO: ISO/ TS 15066:2016 2016). These values are used as a reference in this research, and subsequently the safety evaluation of the HIRC workstation design is performed by comparing the contact forces estimated through the contact model described in this section with respect to the maximum threshold values. It can be noted that the collision model used in this research assumes that the robot controller detects the physical contact with the human and subsequently activates the safety-rated monitored stop function according to ISO (ISO/TS 15066:2016) (2016).

\subsection{Application of method through industrial case}

As a demonstration, the HIRC workstation design process presented in Sect. 3 is applied on a manufacturing workstation from an industrial case.

\subsubsection{Analysis}

A. Investigation of manufacturing workstation The industrial case is from the assembly line of an international heavy-vehicle manufacturing company. The operation includes handling and assembly of a gearbox suspension on the frame of the vehicle. The suspension weighs $8 \mathrm{~kg}$, has an approximate size of $30 \times 20 \times 15 \mathrm{~cm}$ and is currently handled manually at a workstation with a total task time of 7 min (the human operator performs other tasks in addition to the gearbox insulator assembly during this task time). This manual handling of a heavy load implies large biomechanical loads on the human operator. This can be avoided through a HIRC solution where the physically strenuous task of handling the payload is carried out by the robot. Figure 2 presents the current layout of the assembly workstation. The fully manual process begins by the operator carrying the gearbox suspension from position A to position B (position B is a table where pre-assembly is carried out). Subsequently, the gearbox suspension is carried from Position $\mathrm{B}$ and assembled inside the $\mathrm{C}$-shaped frame in position $\mathrm{C}$. The horizontal distance (in X-direction) between $\mathrm{B}$ and $\mathrm{C}$ is approximately 1.8 meters.

B. Requirements specification Operation requirement: The HIRC workstation should enable an assisted material-handling task where a payload of $8 \mathrm{~kg}$ is moved from position A to position $\mathrm{B}$ to position $\mathrm{C}$, with desired orientation.

Performance requirement: Maximum operational time of 7 min and maximum biomechanical load of 4.5 (RULA score) are identified as main performance requirements. 


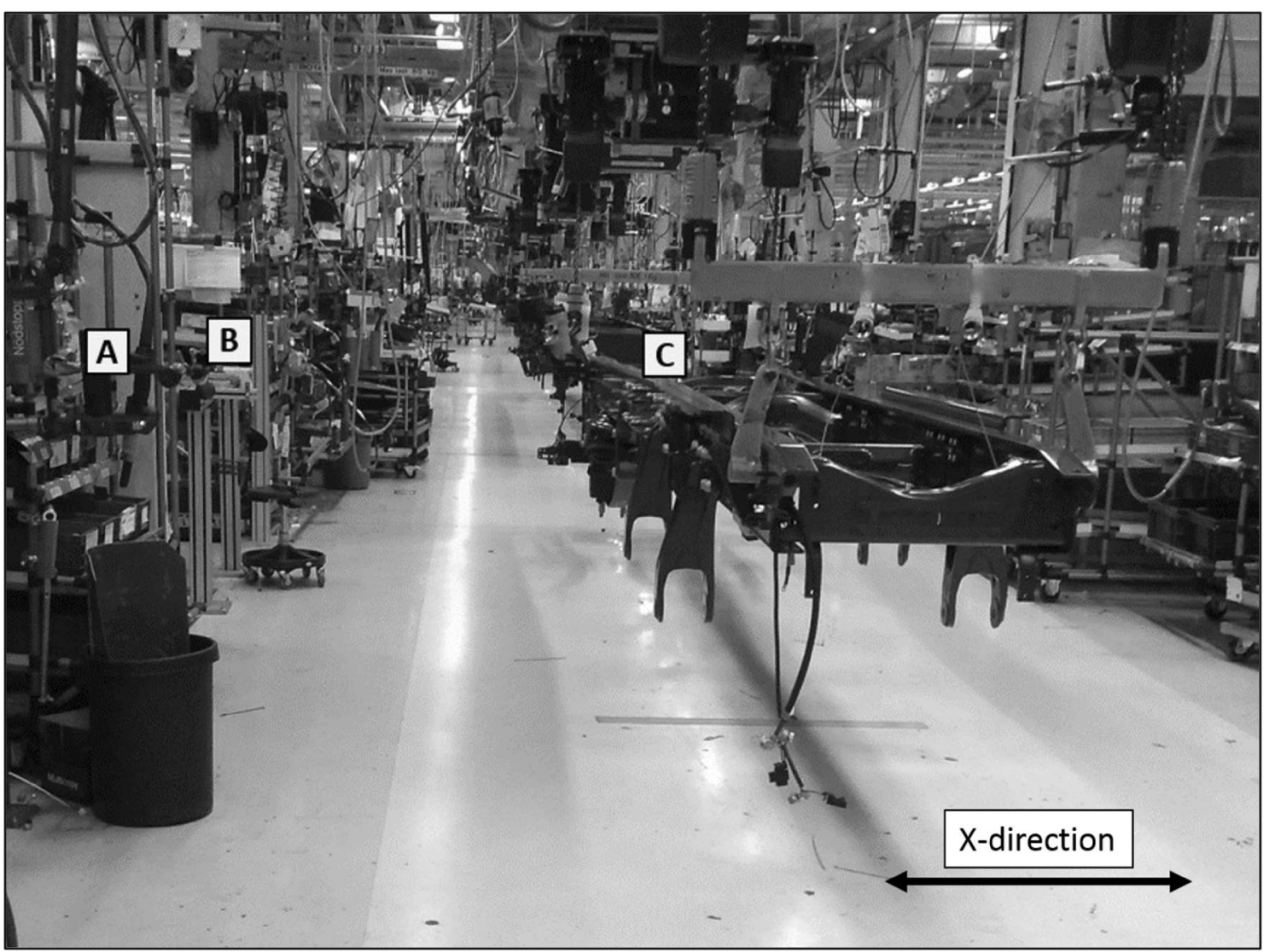

Fig. 2 Existing workstation. The gearbox insulator is moved from the rack (a) to a pre-assembly fixture (b) and finally installed inside the C-shaped frame (c)

4.5 is selected since scores above this value demand urgent investigations and design changes as described in Table 1 (McAtamney and Corlett 1993).

Safety requirement In a HIRC solution, all the body regions of the human operator are at risk of physical collision with the industrial robot. Ideally the safety requirement should be set such that the hypothetical physical collisions that can occur at any point of time during the HIRC operation on any part of the human body should be tolerable, not leading to the onset of pain sensation as specified in ISO/TS 15066 (2016). Since the purpose of this industrial application is limited to the demonstration of the proposed HIRC workstation design process, the safety requirement is limited to constrained transient collisions of the robot with the abdomen region at different points of time during the HIRC operation. Therefore, according to ISO/TS 15066 (2016), a maximum permissible contact force of $110 \mathrm{~N}$ is identified as safety requirement.

C. Task division Task division between human and industrial robot is set at this stage. Table 3 presents the tasks that
Table 3 Task division used in the practical industrial application case

\begin{tabular}{|c|c|c|c|c|}
\hline & Tasks & Pos. in Fig. 2 & Robot & Human \\
\hline 1 & $\begin{array}{l}\text { Move gearbox suspension from } \\
\text { rack }\end{array}$ & A to $B$ & $\mathrm{X}$ & \\
\hline 2 & Preassemble screws & B & & $\mathrm{X}$ \\
\hline 3 & $\begin{array}{l}\text { Move gearbox suspension to } \\
\text { assembly }\end{array}$ & B to $\mathrm{C}$ & $\mathrm{X}$ & \\
\hline 4 & Assemble gearbox suspension & $\mathrm{C}$ & & $X$ \\
\hline
\end{tabular}

are required in this industrial application. Tasks 2 and 4 are difficult to automate due to the complexity of the screws to be pre-assembled and due to uncertainty regarding the position of the frame. On the other hand, tasks 1 and 3 can be executed efficiently by the robot. The resulting task division is presented in Table 3 .

D. Workstation design parameters The HIRC workstation design parameters identified for the industrial case are listed in Table 4. 
Table 4 Workstation design parameters

\begin{tabular}{lll}
\hline Workstation design parameter & Design variable & Dimensions \\
\hline Workstation layout dimensions & & \\
$\begin{array}{l}\text { Human home position } \\
\text { Robot home position }\end{array}$ & $\checkmark$ & Fixed, see Fig. 3a \\
$\begin{array}{l}\text { Incoming material position } \\
\text { Preassembly position }\end{array}$ & & Fixed, see Fig. 3a \\
Frame position & & Fixed, see Fig. 3a \\
Industrial robot & $\checkmark$ & Fixed, see Fig. 3a \\
Robot speed limits & $\checkmark$ & Fixed, ABB IRB 4600 \\
Anthropometrics of the operator & & {$[0.2 \mathrm{~m} / \mathrm{s}, 0.3 \mathrm{~m} / \mathrm{s}]$} \\
Robot gripper design & & Average Swedish [male, female $]^{2}$ \\
Geometrical robot paths & Fixed, see Fig. 3a \\
Handover position between human and & & Fixed, see Fig. 3b \\
robotic tasks & & Fixed, see Fig. 3c \\
\hline
\end{tabular}

${ }^{1}$ Position in X-direction from assembly position in frame (position ' $\mathrm{C}$ ' in Fig. 2)

${ }^{2}$ Anthropometrics from average (with regard to height and weight) male and female body types presented by Hanson et al. (Hanson et al. 2009)

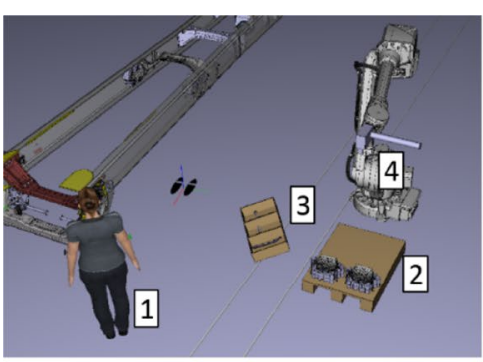

(a)

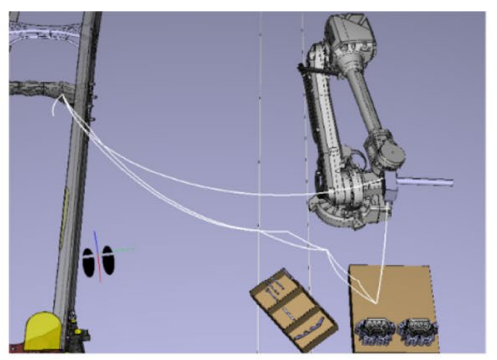

(b)

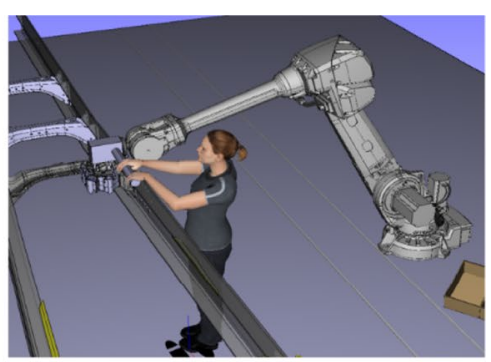

(c)

1. Human home position

2. Incoming material position

3. Preassembly position

4. Robot gripper design

Fig. 3 Visual representation of a human home position, incoming material and pre-assembly position and robot gripper design, $\mathbf{b}$ robot tool centre path, $\mathbf{c}$ handover position

\subsubsection{Synthesis}

E. Selection of design variables In this given case all of the design parameters listed in Table 4 can be considered as design variables for effective design space exploration. Three design parameters were selected: robot home position, robot speed and anthropometrics of the operator. The other design parameters are fixed.

F. Set values on design parameters The HIRC workstation corresponding to the industrial case is designed by dimensioning the parameters and variables as represented in Table 4.

\subsubsection{Evaluation}

Based on the synthesis phase a virtual model of the HIRC workstation is created using the IPS-HIRC software. This model is used to simulate the HIRC process of the industrial case. Screenshots during the execution of this process are presented in Fig. 4. Using this model, evaluation of the HIRC workstation is carried out through performance and safety simulations. This process is described in the rest of this section.

G. HIRC performance and safety simulation The virtual model as presented in Fig. 4 is used to simulate the performance capabilities of the HIRC workstation. Subsequently 


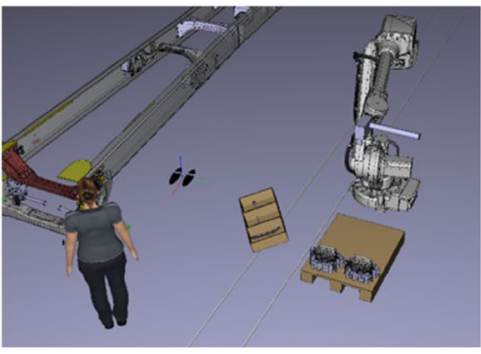

(a)

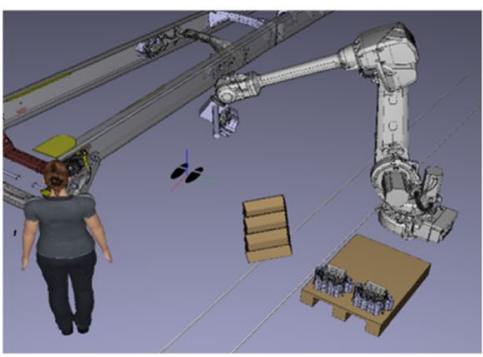

(d)

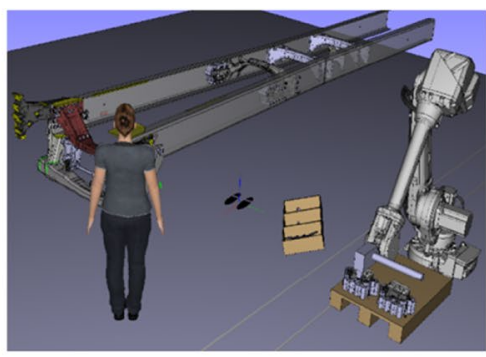

(b)

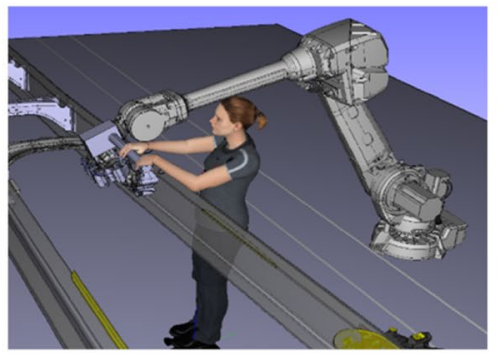

(e)

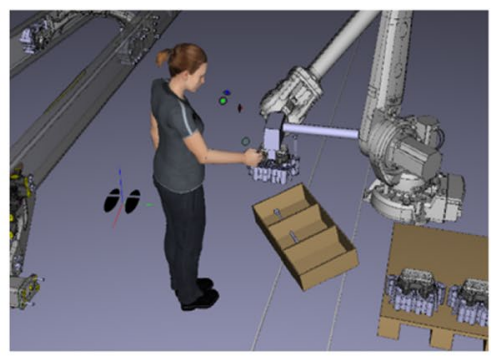

(c)

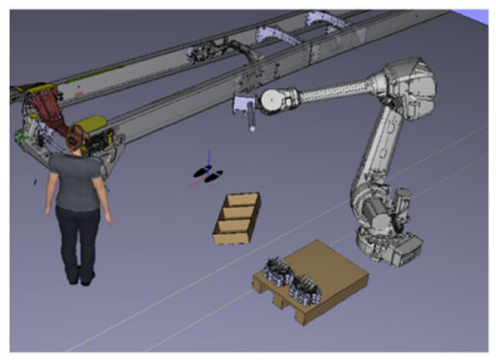

(f)

Fig. 4 a Robot at home position, $\mathbf{b}$ robot at grasp position, $\mathbf{c}$ human does pre-assembly while robot acts as a fixture, $\mathbf{d}$ robot moves to assembly position, e collaborative assembly to final position and gearbox suspension, $\mathbf{f}$ empty robot moves back to home position

Table 5 HIRC workstation design alternatives

\begin{tabular}{llll}
\hline $\begin{array}{l}\text { Design } \\
\text { alternative }\end{array}$ & $\begin{array}{l}\text { Robot speed } \\
(\mathrm{m} / \mathrm{s})\end{array}$ & $\begin{array}{l}\text { Anthropometrics of } \\
\text { the operator }\end{array}$ & $\begin{array}{l}\text { Robot position in } \\
X \text {-direction }(\mathrm{m})\end{array}$ \\
\hline$a$ & 0.2 & Female & 1.8 \\
$b$ & 0.3 & Female & 1.8 \\
$c$ & 0.2 & Male & 1.8 \\
$d$ & 0.2 & Male & 1.4 \\
\hline
\end{tabular}

quantitative data corresponding to biomechanical load (RULA) and operation time(s) are estimated for different design alternatives.

From the definition of robot trajectory in the synthesis phase, inputs to the collision model are derived in terms of the robot's effective mass and maximum impact velocities. Since the safety simulations are intended for a worstcase scenario, constrained transient collisions between the human's abdomen region and the robot are assumed to occur at maximum robot speeds all along the HIRC operation. To perform the simulations, input data corresponding to biomechanical stiffness of the human's abdomen region $(\mathrm{K})$ and effective mass $\left(\mathrm{M}_{\mathrm{H}}\right)$ values are assigned as $10 \mathrm{~N} / \mathrm{mm}$ and $40 \mathrm{~kg}$, respectively, from Table 2 . Furthermore, for the worst-case collision scenario, the human-robot impact is assumed to be highly inelastic by considering the restitution coefficient (CR) at the value of 0.2. Using this input in the collision model, safety simulations are performed to derive maximum collision forces for different design alternatives.

H. HIRC workstation design selection A few design alternatives as presented in Table 5 are considered for demonstrating the process of evaluating the HIRC workstation. Quantitative data corresponding to RULA, operational time and contact forces are measured for each of the design alternatives based on performance and safety simulations. These data are presented in Fig. 5.

In this study, operational time, average RULA score and maximum contact force are used as metrics to measure the performance and safety characteristics of HIRC design alternatives. These metrics are presented in Table 6 and are based on the simulation data presented in Fig. 5.

Based on Table 6 , the most promising design alternative can be selected by comparing the performance and safety metrics with respect to the requirements specifications set in the analysis phase. From a time and biomechanical load perspective all design alternatives meet the requirements. However, none of the design alternatives meet the safety requirements, since the maximum contact forces exceed $110 \mathrm{~N}$ in all of them. Therefore, from this evaluation it can be recommended that the design space needs to be further explored, for instance by relaxing speed limits of the robot or by considering an industrial robot with better safety characteristics such as reduced weight and soft covering to achieve an acceptable HIRC workstation design. 


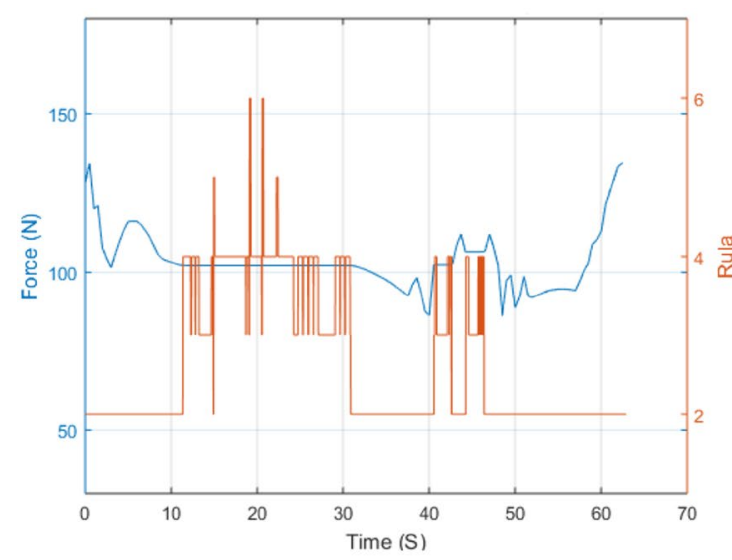

(a)

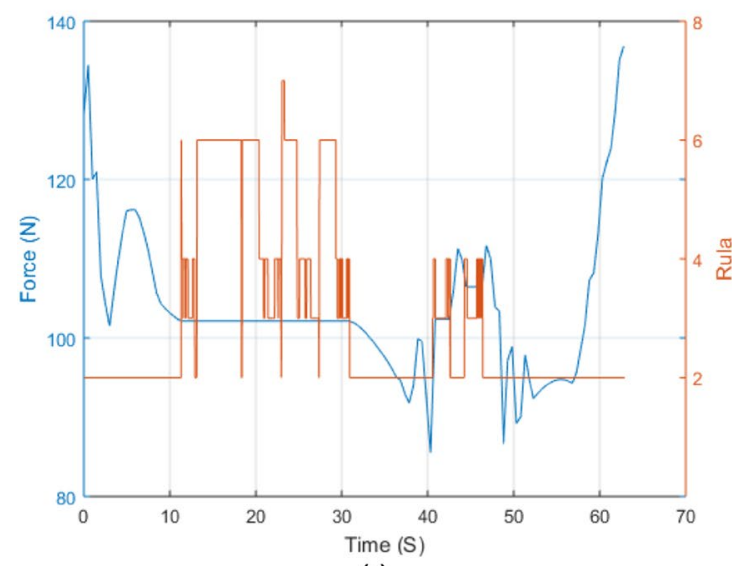

(c)

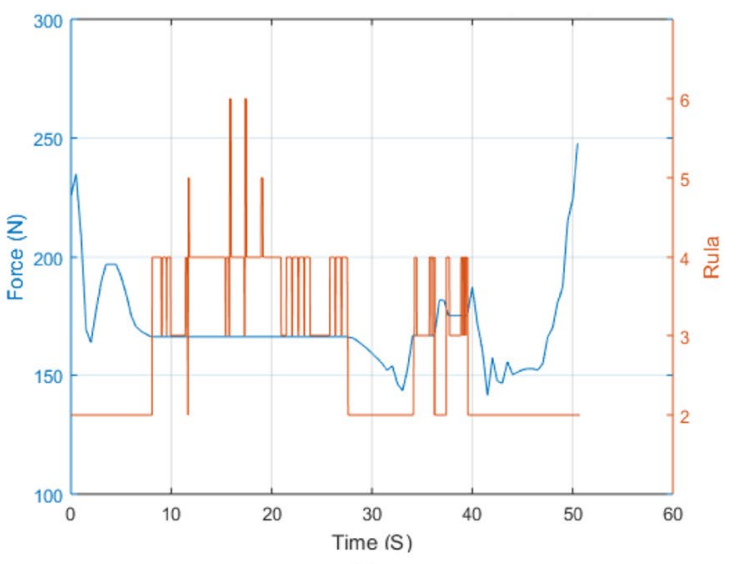

(b)

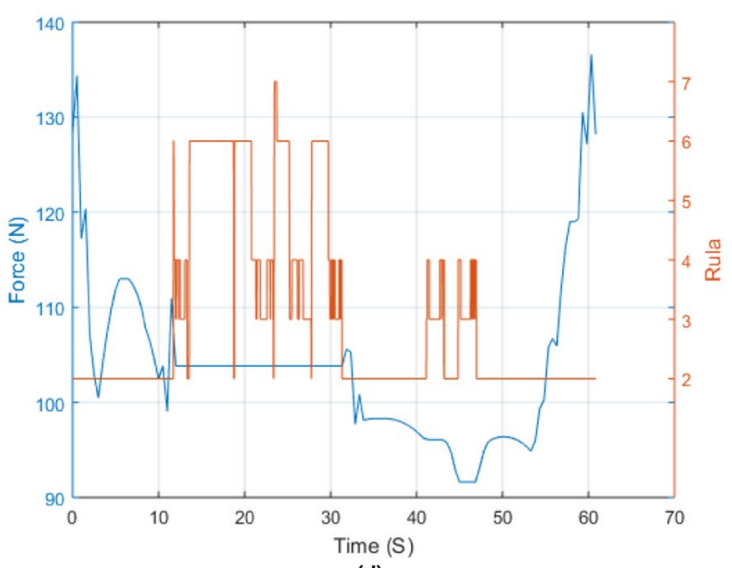

(d)

Fig. 5 HIRC workstation design evaluation of the design alternatives $\mathbf{a}, \mathbf{b}, \mathbf{c}$ and $\mathbf{d}$ for the industrial case

Table 6 Evaluation data for the workstation design cases

\begin{tabular}{llll}
\hline $\begin{array}{l}\text { Design alterna- } \\
\text { tive }\end{array}$ & $\begin{array}{l}\text { Operational } \\
\text { time }\end{array}$ & $\begin{array}{l}\text { Average RULA } \\
\text { score }\end{array}$ & $\begin{array}{l}\text { Maximum } \\
\text { contact force } \\
(\mathrm{N})\end{array}$ \\
\hline$a$ & 62.9 & 3.45 & 134.6 \\
$b$ & 50.7 & 3.49 & 247.7 \\
$c$ & 62.9 & 3.88 & 136.8 \\
$d$ & 60.9 & 3.88 & 136.6 \\
\hline
\end{tabular}

\section{Discussion and conclusions}

This research concerns human-industrial robot collaboration (HIRC) in manufacturing industry. Although this concept is very promising in terms of productivity and profitability, industrial practitioners currently have many apprehensions regarding its applicability in an industrial context, especially due to human safety considerations. In order to address this, several simulation-based methodologies have been presented in the literature to evaluate HIRC workstation design characteristics. These methods either have emphasised the evaluation of performance characteristics (Tsarouchi et al. 2017) or are strictly limited to the human safety aspects in a fenceless environment (Park et al. 2011; Oberer and Schraft 2007). This research contributes by introducing an evaluation methodology that can help industrial practitioners to make better production investment decisions and support a better-grounded decision through evaluation of both performance and safety characteristics during the HIRC workstation design process. The applicability of the proposed evaluation methodology is verified by applying it on an industrial manufacturing case from a heavy-vehicle manufacturing company.

HIRC workstation application designers can benefit from the proposed HIRC workstation evaluation methodology in the following ways:

1. The evaluation method of the workstation design enables the HIRC application designer to carry out risk assessments based on safe human-industrial robot collision behaviour. Furthermore, the HIRC application designer can also use the proposed design process to correlate the 
risks involved with respect to performance, in terms of operational time and biomechanical load, of a specific workstation design. This will facilitate finding the right balance between the human operator's safety and operational performance through HIRC workstations.

2. The evaluation criteria related to operational time, biomechanical load and contact forces are used to identify the most appropriate design for given objectives and requirements of the task. The iteration loops presented in Fig. 1 in the evaluation process are used for this purpose. The same iteration process can also be used to make further design improvements even when all the requirements are met-is it, for example, possible to reduce operation time further by increasing robot speed and still be within the requirements?

3. Most importantly, application designers can use the proposed HIRC workstation evaluation method to make decisions on whether HIRC is indeed the right option for executing a given manufacturing operation.

\subsection{Limitations and future work}

1. It could be noted that the main purpose of this research is targeted towards evaluation of HIRC workstation design alternatives corresponding to a specific combination of workstation design variables. However, the ASE-based HIRC workstation design process used in this research does not include the process of finding an optimal set of workstation design variables in order to achieve the optimal HIRC workstation design by means of finding the ideal trade-offs between safety and performance objectives. Therefore, in future work, an optimisation-based workstation design approach will be integrated into the proposed ASE-based HIRC workstation design process, which can enable the HIRC application designer to further optimise the workstation design through identification of ideal workstation design variables.

2. An experimental verification of the human motions estimated from the software used in this study has not been carried out, which can therefore be considered as a limitation of this research and is being considered for future work for this research.

3. The major emphasis in the proposed research has been that the method application and subsequent use of software to quantify the evaluation criteria should not be userdependent. Therefore, the software used in this research does not include the variation in human and robot motions since the software used is deterministic and produces one optimal path for human and robot. However, this limits the handling of natural deviation of the human worker and hence may exclude some of the worst-case scenarios that could arise during the manufacturing operation.
4. At this stage of the simulation process it will take about $10 \mathrm{~min}$ to assign different values to any number of chosen design parameters and execute the simulation of a HIRC workstation evaluation. Automating this simulation process to make it possible to efficiently specify a large number of design parameters in the HIRC workstation evaluation process will be a future work in this research.

Acknowledgements Open access funding provided by Mälardalen University. The research work was funded by the Swedish Knowledge Foundation (for the INNOFACTURE Research School), Vinnova (for the ToMM2 and Virtual Verification of Human-Robot Collaboration research projects), Scania, ABB and Mälardalen University. The research was conducted in the context of the XPRES research and education environment at Mälardalen University.

Open Access This article is distributed under the terms of the Creative Commons Attribution 4.0 International License (http://creativeco mmons.org/licenses/by/4.0/), which permits unrestricted use, distribution, and reproduction in any medium, provided you give appropriate credit to the original author(s) and the source, provide a link to the Creative Commons license, and indicate if changes were made.

\section{References}

Behrens, R., Elkmann, N.: Study on meaningful and verified thresholds for minimizing the consequences of human-robot collisions. In: 2014 IEEE International Conference on Robotics and Automation (ICRA), pp. 3378-3383 (2014)

Bellgran, M.: Systematic Design of Assembly Systems: Preconditions and Design Process Planning. PhD thesis, Linköping University, Linköping, Sweden (1998)

Bohlin, R., Delfs, N., Hanson, L., Högberg, D., Carlson, J.S.: Automatic creation of virtual manikin motions maximizing comfort in manual assembly processes. In: Technologies and Systems for Asssembly Quality, Productivity and Customization: Proceedings of the 4th CIRP Conference on Assembly Technologies and Systems, pp. 209-212 (2012)

Braha, D., Maimon, O.: The design process: properties, paradigms, and structure. IEEE Trans. Syst. Man Cybern. Part A: Syst. Humans 27(2), 146-166 (1997)

Chen, F., Sekiyama, K., Huang, J., Sun, B., Sasaki, H., Fukuda, T.: An assembly strategy scheduling method for human and robot coordinated cell manufacturing. Int. J. Intell. Comput. Cybern. 4(4), 487-510 (2011)

Dagalakis, N.G., Yoo, J.-M., Oeste, T.: Human-robot collaboration dynamic impact testing and calibration instrument for disposable robot safety artifacts. Ind. Robot: Int. J. 43(3), 328-337 (2016). https://doi.org/10.1108/IR-06-2015-0125

David, G.C.: Ergonomic methods for assessing exposure to risk factors for work-related musculoskeletal disorders. Occup. Med. 55(3), 190-199 (2005)

Flores, P., Ambrósio, J., Claro, J.C.P., Lankarani, H.M.: Influence of the contact-impact force model on the dynamic response of multi-body systems. Proc Inst Mech Eng Part K J Multi-Body Dyn 220(1), 21-34 (2006)

Fryman, J., Matthias, B.: Safety of industrial robots: from conventional to collaborative applications. In: Proceedings of ROBOTIK 2012; 7th German Conference on Robotics, pp. 1-5 (2012) 
Genaidy, A.M., Al-Shedi, A.A., Karwowski, W.: Postural stress analysis in industry. Appl. Ergon. 25(2), 77-87 (1994). https://doi. org/10.1016/0003-6870(94)90068-X

Haddadin, S., Albu-Schaffer, A., Frommberger, M., Rossmann, J., Hirzinger, G.: The "DLR crash report": towards a standard crashtesting protocol for robot safety-part II: Discussions. In: 2009 IEEE International Conference on Robotics and Automation, pp. 280-287 (2009)

Haddadin, S., Albu-Schäffer, A., Hirzinger, G.: Safety evaluation of physical human-robot interaction via crash-testing. In: Robotics: Science and Systems, pp. 217-224 (2007)

Haley, J.: Anthropometry and Mass Distribution for Human Analogues. Volume 1: Military Male Aviators, pp. 33-38. Anthropology Research Project, Yellow Springs, OH (1988) (Haley initiated the project; he did not prepare the final report.)

Hanson, L., Sperling, L., Gard, G., Ipsen, S., Olivares Vergara, C.: Swedish anthropometrics for product and workplace design. Appl. Ergon. 40(4), 797-806 (2009). https://doi.org/10.1016/j.aperg o.2008.08.007

Helms, E., Schraft, R.D., Hagele, M.: rob@work: Robot assistant in industrial environments. In: Proceedings of the 11th IEEE International Workshop on Robot and Human Interactive Communication, pp. 399-404 (2002)

Hertz, H.: On the contact of elastic solids. J. Reine Angew. Math. 92, 156-171 (1881)

Hignett, S., McAtamney, L.: Rapid entire body assessment (REBA). Appl. Ergon. 31(2), 201-205 (2000)

Högberg, D., Hanson, L., Bohlin, R., Carlson, J.S.: Creating and shaping the DHM tool IMMA for ergonomic product and production design. Int. J. Digit. Hum. 1(2), 132-152 (2016)

Huelke, M.O., Ottersbach, H.J: How to approve collaborating robotsthe IFA force pressure measurement system. Paper presented at the 7th International Conference on Safety of Industrial Automated Systems (2012)

Hunt, K.H., Crossley, F.R.E.: Coefficient of restitution interpreted as damping in vibroimpact. J. Appl. Mech. 42(2), 440-445 (1975)

ISO: ISO 10218-2:2011: Robots and robotic devices-safety requirements for industrial robots-Part 2: Robot systems and integration. International Organization for Standardization, Geneva (2011)

ISO: ISO/TS 15066:2016: Robots and Robotic Devices-Collaborative Robots. International Organization for Standardization, Geneva (2016)

Karhu, O., Kansi, P., Kuorinka, I.: Correcting working postures in industry: a practical method for analysis. Appl. Ergon. 8(4), 199-201 (1977)

Kee, D., Karwowski, W.: A comparison of three observational techniques for assessing postural loads in industry. Int. J. Occup. Saf. Ergon. 13(1), 3-14 (2007)

Khatib, O., Burdick, J.: Optimization of dynamics in manipulator design: the operational space formulation. Int. J. Robot. Autom. 2(2), 90-98 (1987)

Krüger, J., Nickolay, B., Heyer, P., Seliger, G.: Image based 3D surveillance for flexible man-robot-cooperation. CIRP Ann. Manuf. Technol. 54(1), 19-22 (2005). https://doi.org/10.1016/s0007 -8506(07)60040-7

Krüger, J., Lien, T.K., Verl, A.: Cooperation of human and machines in assembly lines. CIRP Ann. Manuf. Technol. 58(2), 628-646 (2009). https://doi.org/10.1016/j.cirp.2009.09.009

Laring, J., Forsman, M., Kadefors, R., Örtengren, R.: MTM-based ergonomic workload analysis. Int. J. Ind. Ergon. 30(3), 135-148 (2002). https://doi.org/10.1016/S0169-8141(02)00091-4

Luckman, J.: An approach to the management of design. J. Oper. Res. Soc. 18(4), 345-358 (1967)

Maeno, T., Hasegawa, J.: Development of a finite element model of the total human model for safety (THUMS) and application to car-pedestrian impacts. In: Proceedings of the 17th International Technical Conference on the Enhanced Safety of Vehicles, pp. 1-10 (2001)

Matthias, B., Oberer-Treitz, S., Ding, H.: Experimental characterization of collaborative robot collisions. In: ISR/Robotik 2014; 41st International Symposium on Robotics, pp. 1-6 (2014)

Maynard, H.B., Stegemerten, G.J., Schwab, J.L.: Methods Time Measurement. McGraw-Hill, New York (1948)

McAtamney, L., Corlett, E.N.: RULA: a survey method for the investigation of work-related upper limb disorders. Appl. Ergon. 24(2), 91-99 (1993)

Michalos, G., Makris, S., Tsarouchi, P., Guasch, T., Kontovrakis, D., Chryssolouris, G.: Design considerations for safe human-robot collaborative workplaces. Proc. CIRP 37, 248-253 (2015)

Oberer, S., Schraft, R.D.: Robot-dummy crash tests for robot safety assessment. In: Proceedings 2007 IEEE International Conference on Robotics and Automation, pp. 2934-2939 (2007)

Oberer, S., Schraft, R.D.: Robot-dummy crash tests for robot safety assessment. In: Proceedings-2007 IEEE International Conference on Robotics and Automation, pp. 2934-2939 (2007)

Oberer-Treitz, S., Puzik, A., Verl, A.: Measuring the collision potential of industrial robots. In: ISR 2010 (41st International Symposium on Robotics) and ROBOTIK 2010 (6th German Conference on Robotics), pp. 1-7 (2010)

Ore, F., Hanson, L., Wiktorsson, M., Eriksson, Y.: Automation constraints in human-industrial robot collaborative workstation design. Paper presented at the 7th International Swedish Production Symposium (2016)

Ore, F., Hanson, L., Delfs, N., Wiktorsson, M.: Human industrial robot collaboration-development and application of simulation software. Int. J. Hum. Factors Model. Simul. 5(2), 164-185 (2015)

Park, J.-J., Haddadin, S., Song, J.-B., Albu-Schäffer, A.: Designing optimally safe robot surface properties for minimizing the stress characteristics of human-robot collisions. In: 2011 IEEE International Conference on Robotics and Automation, pp. 5413-5420 (2011)

Pini, F., Leali, F., Ansaloni, M.: A systematic approach to the engineering design of a HRC workcell for bio-medical product assembly. In: 2015 IEEE 20th Conference on Emerging Technologies and Factory Automation (ETFA), pp. 1-8 (2015)

Povse, B., Koritnik, D., Bajd, T., Munih, M.: Correlation between impactenergy density and pain intensity during robot-man collision. In: 3rd IEEE RAS and EMBS International Conference on Biomedical Robotics and Biomechatronics (BioRob), pp. 179-183 (2010)

Robin, S.: HUMOS: human model for safety-a joint effort towards the development of refined human-like car occupant models. In: Proceedings of the 17th International Technical Conference on the Enhanced Safety of Vehicles, p. 297 (2001)

IPS: IPS IMMA. http://industrialpathsolutions.se/ips-imma/ (2017). Accessed 2 Dec 2017

Segeborn, J., Segerdahl, D., Ekstedt, F., Carlson, J.S., Andersson, M., Carlsson, A., Söderberg, R.: An industrially validated method for weld load balancing in multi station sheet metal assembly lines. J. Manuf. Sci. Eng. 136(1), 011002 (2014)

Tan, J.T.C., Duan, F., Zhang, Y., Watanabe, K., Kato, R., Arai, T.: Human-robot collaboration in cellular manufacturing: design and development. In: IROS 2009. Proceedings of the 2009 IEEE/RSJ International Conference on Intelligent Robots and Systems, pp. 29-34 (2009)

Thomas, C., Stankiewicz, L., Grötsch, A., Wischniewski, S., Deuse, J., Kuhlenkötter, B.: Intuitive work assistance by reciprocal humanrobot interaction in the subject area of direct human-robot collaboration. Proc. CIRP 44, 275-280 (2016)

Tsarouchi, P., Makris, S., Chryssolouris, G.: Human-robot interaction review and challenges on task planning and programming. Int. J. Comput. Integr. Manuf. 29(8), 916-931 (2016a)

Tsarouchi, P., Spiliotopoulos, J., Michalos, G., Koukas, S., Athanasatos, A., Makris, S., Chryssolouris, G.: A decision making 
framework for human robot collaborative workplace generation. Proc. CIRP 44, 228-232 (2016b)

Tsarouchi, P., Matthaiakis, A.-S., Makris, S., Chryssolouris, G.: On a human-robot collaboration in an assembly cell. Int. J. Comput. Integr. Manuf. 30(6), 580-589 (2017)

Unfallversicherung, D.G.: BG/BGIA risk assessment recommendations according to machinery directive: design of workplaces with collaborative robots. BGIA-Institute for Occupational Safety and Health of the German Social Accident Insurance, Sankt Augustin, Germany (2009)

Vemula, B., Matthias, B., Ahmad, A.: A design metric for safety assessment of industrial robot design suitable for power-and forcelimited collaborative operation. Int. J. Intell. Robot. Appl. 2(2), 226-234 (2018)

Vignais, N., Bernard, F., Touvenot, G., Sagot, J.C.: Physical risk factors identification based on body sensor network combined to videotaping. Appl. Ergon. 65, 410-417 (2017)

Wassink, M., Stramigioli, S.: Towards a novel safety norm for domestic robotics. In: 2007 IEEE/RSJ International Conference on Intelligent Robots and Systems, pp. 3354-3359 (2007)

Wu, B.: Manufacturing Systems Design and Analysis: Context and Techniques, 2nd edn. Chapman \& Hall, London (1994)

Yamada, Y., Hirasawa, Y., Huang, S., Umetani, Y., Suita, K.: Humanrobot contact in the safeguarding space. IEEE/ASME Trans. Mechatron. 2(4), 230-236 (1997)

Zinn, M., Roth, B., Khatib, O., Salisbury, J.K.: A new actuation approach for human friendly robot design. Int. J. Robot. Res. 23(4-5), 379-398 (2004)

Publisher's Note Springer Nature remains neutral with regard to jurisdictional claims in published maps and institutional affiliations.

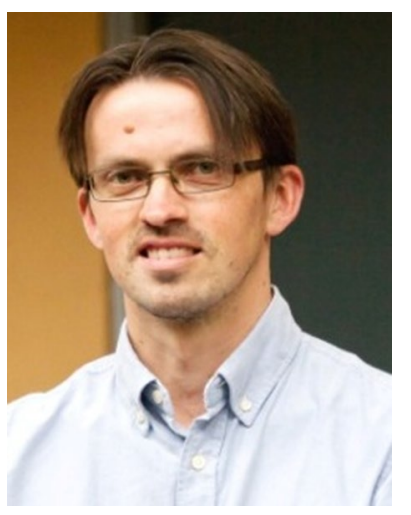

Fredrik Ore is an industrial $\mathrm{PhD}$ student in the research area of Innovation and Product Realisation at Mälardalen University in Eskilstuna, Sweden. His research is in the field of simulation, evaluation and optimisation of human-industrial robot collaboration. He has a Master of Science in Mechanical Engineering from Luleå University of Technology and has been a doctoral student at the Innofacture Research School at Mälardalen University since late 2012.

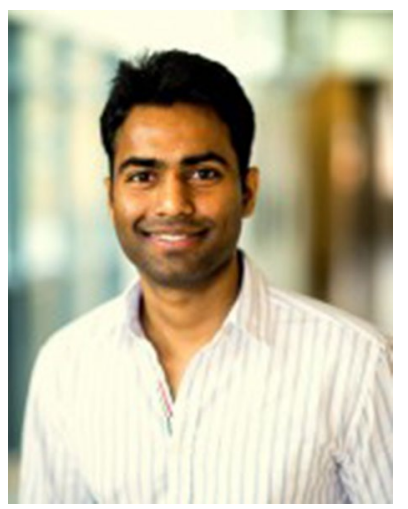

Bhanoday Vemula is a Ph.D. student within the research area of innovation and product realization at Mälardalen University. Bhanoday received his M.Sc degree in Mechanical engineering from Linköping University. He performs research in the area of safety evaluation and design of industrial robots for collaborative manufacturing applications.

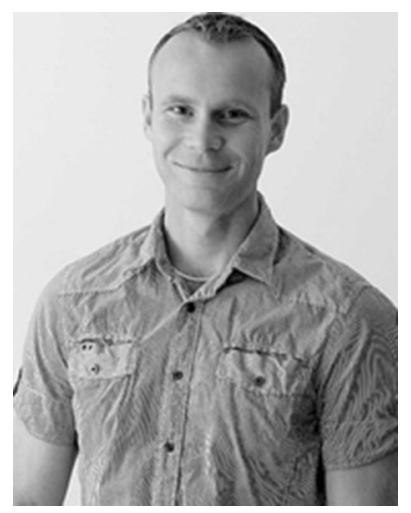

Lars Hanson is professor of Product and Production Development at the School of Engineering Science, Skövde University. His research interest is human machine interaction with special focus on digital human modelling. Hanson is also managing the activities at Scanias Smart Factory Lab, a lab there technologies and processes are adapted, evaluated and demonstrated.

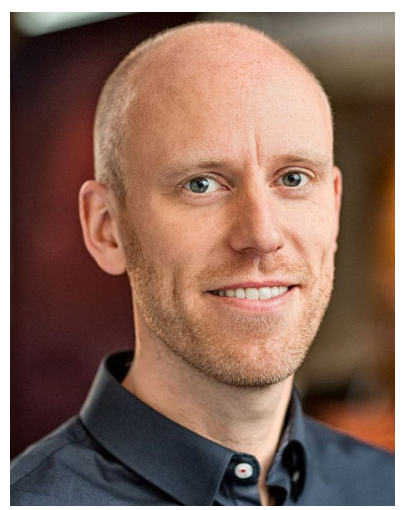

Magnus Wiktorsson is professor of Production Logistics at the department for Sustainable Production Development, at KTH Royal Institute of Technology. $\mathrm{He}$ is also visiting professor at Mälardalen University. His research interest concerns how complex production and logistic systems can be described and predicted, especially considering industrial digitisation and sustainability.

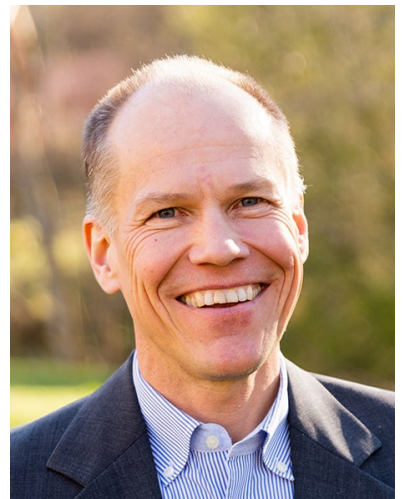

Björn Fagerström presently work as Professor in Product Development at Mälardalen University, Eskilstuna, Sweden. He obtained his $\mathrm{PhD}$ degree in Product Development 2004 at Chalmers University of Technology, Göteborg, Sweden. He combines his work as professor with work as management consultant, mainly related to R\&D Development and industrialization. 